\title{
Multiple eccrine spiradenoma in a young Greek patient: case report and review of the literature
}

\author{
Maria Gerochristou, Maria Mazioti, Theodora Douvali, Maria Gerodimou, Vasiliki Chasapi
}

\author{
Andreas Syggros Hospital of Cutaneous \& Venereal Diseases, Athens, Greece \\ Corresponding author: Dr. Maria Mazioti, E-mail: marakimazioti@gmail.com
}

\begin{abstract}
Eccrine spiradenoma is an uncommon and benign adnexal tumor characterized by one or more nodules. The present article refers to a young patient with multiple and gradually enlarged nodules which remained undiagnosed and untreated for many years. Lesions were initially considered as multiple leiomyomas whereas skin biopsy revealed the existence of uncommon and benign tumors of eccrine and apocrine grandular adnexa confirming the diagnosis of eccrine spiradenoma. Similarities in clinical appearance and symptoms between tumors may lead to erroneous diagnosis and skin biopsy is an essential tool for differential diagnosis.
\end{abstract}

Key words: Tumor; Eccrine glands; Pain

\section{INTRODUCTION}

Eccrine spiradenoma (ES) represents a rare and benign tumor of sweat glands which was first described in 1956 by Kersting and Helwing. The deep dermal position of the tumor is indicative for its origination from the secretory coil of eccrine glands, even though several lines of evidence also support its possible apocrine differentiation $[1,2]$. The aim of this article is to present a rare case of a young Greek patient who was diagnosed with multiple ES.

\section{CASE REPORT}

A 33-year-old Caucasian woman presented to the outpatient clinic of a dermatological center complaining of multiple and gradually enlarged lesions on the right side of the back. The onset of the lesions was observed at the age of 17 years old. Personal and family medical history was negative for comorbidities except from an excision of a giant cell tumor of tendon sheaths in her right arm one year ago. In addition, anaemia was identified after the performance of blood biochemical test.

Physical examination revealed well defined, round to oval, pink nodules. Their size ranged from 0.5 to $1 \mathrm{~cm}$ and they were arranged in linear pattern. Nodules were painful, tender to palpation, firm and fixed to the skin (Figs. 1 and 2).

Histopathological analysis revealed benign tumors of eccrine and apocrine grandular adnexa of the skin. Morphological features of the tumors were typical to eccrine spiradenoma and nodules were extended to the subcutaneous tissue. Immunohistochemical analysis showed strong expression of cytokeratin 7 (CK7), p40 and $\mathrm{p} 63$ and weak expression of the transcriptional factor GATA-3 and carcinoembryonic antigen (CEA). Tumor cells were negative for epithelial membrane antigen (EMA), protein S-100 and gross cystic disease fluid protein 15 (GCDFP-15).

\section{DISCUSSION}

ES is an uncommon, benign adnexal tumor characterized by one or more nodules. The tumor has been described as "blue ball in the dermis" because of its blue color, although it might be purple, red, pink or gray [3]. Few clinical variants have been described like solitary ES which is generally accepted as the most common, multiple ES and giant vascular

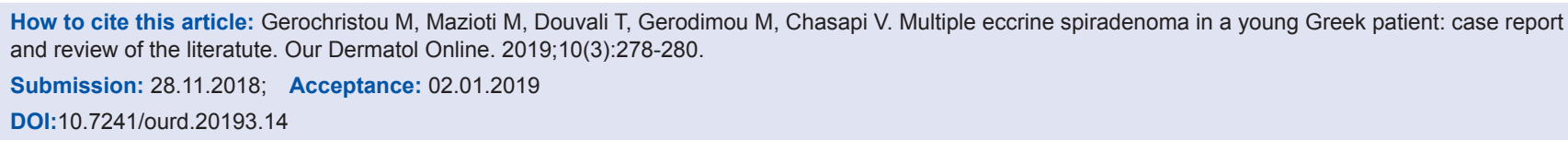




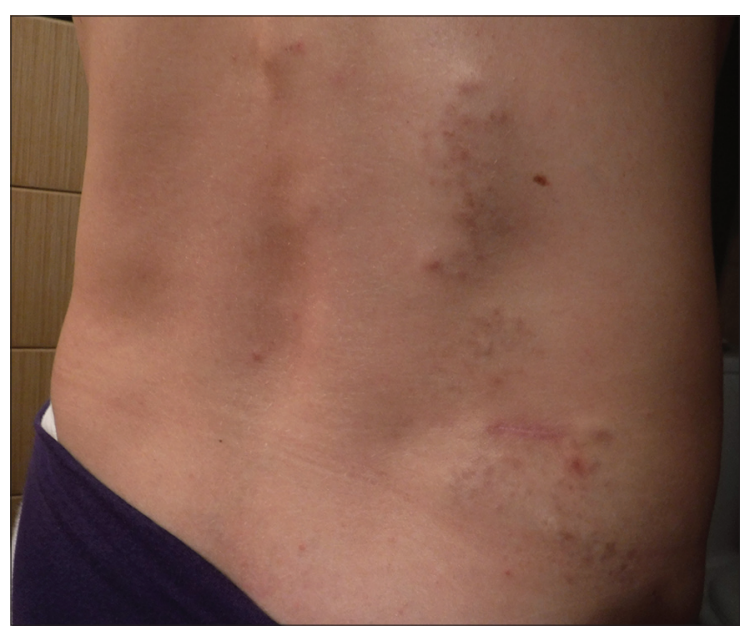

Figure 1: Well defined, round to oval, pink nodules, ranging from 0.5 to $1 \mathrm{~cm}$ and arranged in linear pattern.

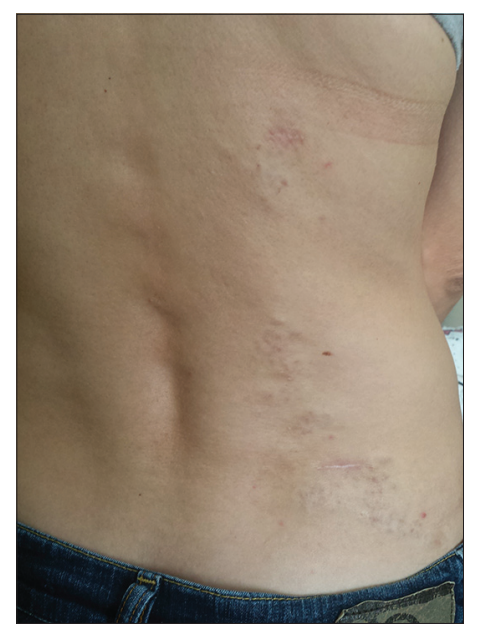

Figure 2: Clinical appearance of lesions after surgical excision.

ES. Review of the literature demonstrates that multiple ES includes less than 2\% of all cases and the majority of these tumors are distributed by linear or zosteriform pattern [4]. Pathways underlying the pathogenesis of the tumor are still being elucidated. The potential role of hereditary factors was pointed out by some investigators who suggested autosomal and dominant inheritance but their observations are still not established [5]. Interestingly, trauma has been speculated as precipitating factor leading to the development of ES [6].

Solitary ES occurs more frequently in men, whereas multiple ES appear more commonly in females, with an estimated female to male ratio of 3:1 [7]. The onset of the tumor is typically observed during the second to fourth decade of life. Solitary ES mostly occurs on the upper half of the body, whereas the distribution of multiple ES involves face, neck and lower extremities.
The main symptom and clinical sign is pain and tenderness to palpation respectively [8]. In our patient lesions were initially observed at the second decade of life but they remained undiagnosed and untreated due to their size and lack of symptoms. Their progressive augmentation resulted in pain which motivated the patient to request medical advice.

Diagnosis is established by skin biopsy which reveals the concomitant presence of large, closely packed cells at the central part of the tumor and smaller cells with compact nucleus at the circumference. Immunochemistry shows expression of CK, CEA, EMA, and S-100 by the tumor cells $[9,10]$.

Surgical excision of the tumor is the most effective treatment option but it is completely impractical for extensive solitary and multiple ES. Radiotherapy or $\mathrm{CO}_{2}$ laser are alternative choices which have shown satisfactory results. Intralesional infusion of botulinum toxin A and triamcinolone has been tested providing minimal improvement and its long term efficacy remains questionable $[11,12]$. It is widely accepted that future studies are needed and until then surgical excision remains the gold standard therapy. The present patient was referred to plastic surgeon and was scheduled for staged excision of tumors. Figure 2 shows the postoperative condition.

\section{CONCLUSION}

The presentation of our case report is interesting from the standpoint that tumors were initially considered as multiple leiomyomas which are also rare and benign neoplasms but they arise from smooth muscle cells. In order to design the appropriate therapeutic strategy, our clinical diagnosis needed to be confirmed by a skin biopsy, which showed the existence of multiple eccrine spiradenoma. In conclusion the present article points out that similarities in clinical appearance and symptoms between tumors may lead to erroneous diagnosis and that skin biopsy is essential for differential diagnosis.

\section{Consent}

The examination of the patient was conducted according to the Declaration of Helsinki principles.

\section{REFERENCES}

1. Singh K, Chatterjee T. Eccrine spiradenoma: A rare adnexal tumor. Indian J Cancer. 2017;54:695. 
2. Dhua S, Sekhar DR. A rare case of eccrine spiradenoma - treatment and management. Eur J Plast Surg. 2016;39:143-6.

3. Salim S, Bounniyt H, El Amraoui M, Benzekri A, Senouci K, Hassam B. Malignant transformation of a spiradenoma with blaschkoïd pattern. Clin Case Rep. 2018;6:2086-8.

4. Ren F, Hu Z, Kong Q, Sang H. Multiple segmental eccrine spiradenoma with a zosteriform pattern: a case report and literature review. Ann Dermatol. 2015;27:435-8.

5. Yoshida A, Takahashi K, Maeda F, Akasaka T. Multiple vascular eccrine spiradenomas: a case report and published work review of multiple eccrine spiradenomas. J Dermatol. 2010;37:990-4.

6. Ren F, Hu Z, Kong Q, Sang H. Multiple Segmental Eccrine Spiradenoma with a Zosteriform Pattern: A Case Report and Literature Review. Ann Dermatolo. 2015;27:435-8.

7. Yoshida A, Takahashi K, Maeda F, Akasaka T. Multiple vascular eccrine spiradenomas: a case report and published work review of multiple eccrine spiradenomas. J Dermatol. 2010;37:990-4.

8. Englander L, Emer JJ, McClain D, Amin B, Turner RB. A rare case of multiple segmental eccrine spiradenomas. J Clin Aesthet
Dermatol. 2011;4:38-44.

9. Sharma A, Sengupta P, Das AK, Nigam MK, Chattopadhya S. Eccrine spiradenoma in knee. Indian J Dermatol. 2014;59:513-5.

10. Rekhi B, Agarwal A. Cytopathologic features of an unusual case of multiple eccrine spiradenomas misdiagnosed as a malignant round cell tumor. J Cytol. 2017;34:39-42.

11. Ren F, Hu Z, Kong Q, Sang H. Multiple Segmental Eccrine Spiradenoma with a Zosteriform Pattern: A Case Report and Literature Review. Ann Dermatol. 2015;27:435-8.

12. Gordon S, Styron BT, Haggstrom A. Pediatric segmental eccrine spiradenomas: a case report and review of the literature. Ped Dermatol. 2013;30:e285-6.

Copyright by Maria Gerochristou, et al. This is an open-access article distributed under the terms of the Creative Commons Attribution License, which permits unrestricted use, distribution, and reproduction in any medium, provided the original author and source are credited.

Source of Support: Nil, Conflict of Interest: None declared. 